\title{
Bacteriological Profile and Drug Resistance Patterns of Blood Culture Isolates in a Tertiary Care Nephrourology Teaching Institute
}

\author{
Kalpesh Gohel, Amit Jojera, Shailesh Soni, Sishir Gang, \\ Ravindra Sabnis, and Mahesh Desai \\ Muljibhai Patel Urological Hospital and Society for Research in Nephro-Urology, Dr. V. V. Desai Road, \\ Nadiad, Gujarat 387001, India \\ Correspondence should be addressed to Kalpesh Gohel; gohelk2000@yahoo.co.in
}

Received 29 December 2013; Revised 23 February 2014; Accepted 13 March 2014; Published 7 April 2014

Academic Editor: Vidya Mave

Copyright (C) 2014 Kalpesh Gohel et al. This is an open access article distributed under the Creative Commons Attribution License, which permits unrestricted use, distribution, and reproduction in any medium, provided the original work is properly cited.

Blood stream infections can lead to life threatening sepsis and require rapid antimicrobial treatment. The organisms implicated in these infections vary with the geographical alteration. Infections caused by MDR organisms are more likely to increase the risk of death in these patients. The present study was aimed to study the profile of organisms causing bacteremia and understand antibiotic resistance patterns in our hospital. 1440 blood samples collected over a year from clinically suspected cases of bacteremia were studied. The isolates were identified by standard biochemical tests and antimicrobial resistance patterns were determined by CLSI guidelines. Positive blood cultures were obtained in 9.2\% of cases of which Gram-positive bacteria accounted for 58.3\% of cases with staph aureus predominance; gram negative bacteria accounted for $40.2 \%$ with enterobactereciea predominence; and $1.5 \%$ were fungal isolates. The most sensitive drugs for Gram-positive isolates were vancomycin, teicoplanin, daptomycin, linezolid, and tigecycline and for Gram-negative were carbapenems, colistin, aminoglycosides, and tigecycline. The prevalence of MRSA and vancomycin resistance was $70.6 \%$ and $21.6 \%$, respectively. ESBL prevalence was $39.6 \%$. Overall low positive rates of blood culture were observed.

\section{Introduction}

Blood stream infections range from self-limiting infections to life threatening sepsis that requires rapid and aggressive antimicrobial treatment [1]. A wide spectrum of organisms has been described that cause blood stream infections and this spectrum is subject to geographical alteration [2-5]. Increasing antimicrobial resistance is a worldwide concern. The prevalence of resistance of blood borne isolates is increasing and it also varies in accordance with geographical and regional location. The infection caused by MDR organisms is more likely to prolong the hospital stay, increase the risk of death, and require treatment with more expensive antibiotics. In almost all cases, antimicrobial therapy is initiated empirically before the results of blood culture are available. Keeping in mind the high mortality and morbidity associated with septicemia, right choice of empiric therapy is of importance [6]. Therefore, the present study was undertaken to analyze the various organisms causing septicemia and their antibiotic resistance patterns, as it would be a useful guide for clinicians initiating the empiric antibiotic therapy.

\section{Materials and Methods}

A total of 1440 samples from clinically suspected cases of bacteremia were studied at Muljibhai Patel Urological Hospital for a period of one year from October 2012 to September 2013. Our institute is 140-bedded teaching hospital which caters to all kinds of nephrology and urology patients including moderate size of hemodialysis programme as well as kidney transplant programme. All the samples were collected from indoor patients in our hospital during the study period and processed in the central laboratory.

Blood was collected from 2 different sites (avg. $8 \mathrm{~mL}$ per site) 20 minutes apart in every patient using strict aseptic 
precautions and inoculated immediately into BacT/ALERT FA plus-aerobic blood culture bottles with $0.025 \%$ of sodium polyanethol sulfonate as anticoagulant. In pediatric cases 1-2 mL of blood was inoculated in BacT/ALERT PF plus pediatric blood culture bottles. After collection these bottles were immediately incubated in BacT/ALERT 3D (manufactured by bioMerieoux) - a fully automated blood culture system for detection of growth in blood culture. The negative results were followed up to 7 days and final report was issued. While, in case of a positive growth, the BacT/ALERT automatically gives an alert. The positive bottles were then subcultured on chrome agar. From the colonies on chrome agar $0.5 \mathrm{McF}$ arland suspension was prepared which was then subjected to identification and susceptibility testing on Mini API $(n=50)$ till February 2013 or Vitek $2(n=82)$ from March 2013 onwards (manufactured by bioMerieoux) - which is a fully automated system for identification of organism and antimicrobial susceptibility testing as per the CLSI 2013 guidelines. The ESBL status was determined by Mini API/Vitek 2 as per the CLSI guidelines and was not subjected to any further testing.

\section{Results}

During the study period, 1440 blood cultures were analyzed of which 132 microorganisms were isolated, out of which 130 were bacterial isolates and 2 were fungal isolate, that is, Candida albicans. Their mean age was $48.6 \pm 14.8$ years of which 89 were males and 43 were females. During study period we did not observe multiple positive blood cultures from any patient. The distribution and percentage of various bacterial and fungal isolates are shown in Table 1.

Among the Gram-positive isolates, the predominant isolate was Staphylococcus aureus as shown in Table 2 which exhibited least resistance to tetracycline, doxycycline, vancomycin, teicoplanin, tigecycline, daptomycin, and linezolid. Oxacillin resistance (MRSA) was $70.6 \%$ in these strains. Vancomycin resistance in Staphylococcus aureus isolates was 21.6\%. The vancomycin intermediate Staphylococcus aureus was observed in 1 patient. In VRSA strains the MIC for vancomycin was $\geq 32$. This was not confirmed further by reference MIC testing.

Other Gram-positive isolates coagulase negative staphylococcal strains (CONS) showed least resistance to gentamicin, quinolones, co-trimoxazole, tigecycline, linezolid, and tetracycline. Kocuria rosea showed no resistance to tetracycline and co-trimoxazole while Micrococcus showed least resistance to tetracycline, vancomycin, tigecycline, and levofloxacin. Enterococci showed least resistance to tetracycline, teicoplanin, and tigecycline. Streptococcus was isolated in one case only.

Among the Gram-negative isolates, the predominant isolates were E. coli and Klebsiella in 33 of $53(62.3 \%)$ as highlighted in Table 3 of which 21 (39.6\%) were ESBL producers. E. coli isolates showed least resistance to carbapenems, aminoglycosides, and tigecycline and moderate resistance to beta-lactam beta-lactamase inhibitors. Klebsiella showed least resistance to carbapenems and moderate
TABLE 1: Distribution of isolates in blood cultures.

\begin{tabular}{lcc}
\hline Type & Numbers & Percentage \\
\hline Staphylococcus & 51 & 38.6 \\
E. coli & 20 & 15.2 \\
Klebsiella & 13 & 9.8 \\
Pseudomonas & 7 & 5.3 \\
Kocuria & 7 & 5.3 \\
Micrococcus & 7 & 5.3 \\
Burkholderia & 6 & 4.5 \\
Coagulase-negative staphylococci & 6 & 4.5 \\
Enterococcus & 5 & 3.8 \\
Sphingomonas & 4 & 3.0 \\
Candida & 2 & 1.5 \\
Acinetobacter & 2 & 1.5 \\
Moraxella & 1 & 0.8 \\
Streptococcus & 1 & 0.8 \\
\hline
\end{tabular}

resistance to aminoglycosides, tigecycline, and beta-lactam beta-lactamase inhibitor combination. Pseudomonas showed least resistance to carbapenems, piperacillin-tazobactam, and aminoglycosides. Other Gram-negative isolates were Burkholderia in 6 cultures, Sphingomonas in 4, Acinetobacter in 2, and Moraxella in 1 culture. Eight isolates including 2 CONS, 2 Micrococcus, 2 Sphingomonas, 1 Burkholderia, and 1 Moraxella were considered contaminated based on clinical and supporting laboratory indicators.

\section{Discussion}

In the present paper, blood culture positivity was seen in 132 of $1440(9.2 \%)$ cases which is quite similar to Mehta et al. [7] and China and Gupta [8] but quite lower to other studies of Kamga et al. [9], Kavitha et al. [10], and Roy et al. [11]. We feel the low incidence in our paper is due to various reasons. Majority of the patients reported to us are referred by other specialists or hospitals and these patients were offered antibiotics elsewhere before they reached our hospital. Many patients developed infections after hospitalization or after surgery by which they already had been given antibiotics before sampling of blood for culture.

The incidence of Gram-positive organisms was 77/132 (58.3\%) while 53/132 (40.2\%) were Gram-negative isolates in our paper. It is in accordance with the studies of China and Gupta [8], Kamga et al. [9], Anbumani et al. [12], and Karlowsky et al. [13] who reported similar incidencesbut in most of the studies like Mehta et al. [7], Mehdinejad et al. [14], Barati et al. [15], and Ayobola et al. [16] Gramnegative organisms have taken over Gram-positive organisms in hospital settings. This difference could be related to an active dialysis programme and substantial contribution of dialysis line or catheter related infections which are usually of Gram-positive nature. This also indicates that infections by Gram-positive organisms constitute a significant threat to septicemia in our locale and the spectrum of organisms is subject to geographical alterations. 
TABLE 2: Drug resistance pattern of major Gram-positive isolates.

\begin{tabular}{|c|c|c|c|c|}
\hline \multirow{2}{*}{ Antimicrobial tested } & \multicolumn{2}{|c|}{ Enterococcus $(n=5)$} & \multicolumn{2}{|c|}{ Staphylococcus aureus $(n=51)$} \\
\hline & Mini API $(n=3)$ & Vitek $(n=2)$ & Mini API $(n=21)$ & Vitek $(n=30)$ \\
\hline Amoxicillin & NP & NP & 100 & 100 \\
\hline Cefotaxime + clavulanate & NP & 50 & NP & 37 \\
\hline Ceftazidime + clavulanate & NP & 50 & NP & 37 \\
\hline Cefepime + tazobactam & NP & 50 & NP & 30 \\
\hline Tigecycline & NP & 0 & NP & NP \\
\hline Gentamycin & 33 & 50 & NP & NP \\
\hline Amikacin & 33 & 50 & NP & NP \\
\hline Ciproflox & 100 & 100 & 90 & 87 \\
\hline Levoflox & 100 & 100 & 75 & 80 \\
\hline Nalidixic acid & 100 & 100 & NP & NP \\
\hline Nitrofurantoin & 100 & 100 & 0 & 78 \\
\hline Tetracycline & 33 & 50 & 10 & 27 \\
\hline Doxycycline & 33 & 50 & 10 & 27 \\
\hline Minocycline & 33 & 50 & 10 & 27 \\
\hline Oxacillin & NP & NP & 73 & 67 \\
\hline Vancomycin ${ }^{* *}$ & 50 & 50 & 20 & 22 \\
\hline Teicoplanin & 33 & 50 & NP & 0 \\
\hline Daptomycin & NP & 0 & NP & 6 \\
\hline Linezolid & NP & 0 & NP & 3 \\
\hline
\end{tabular}

NP: drug not in panel; ${ }^{* *}$ see Section 4.

Figures in the table are expressed in percentages.

TABLE 3: Drug resistance pattern of major Gram-negative isolates.

\begin{tabular}{|c|c|c|c|c|c|}
\hline \multirow{2}{*}{ Antimicrobial tested } & \multicolumn{2}{|c|}{ E. $\operatorname{Coli}(n=20)$} & \multirow{2}{*}{$\begin{array}{c}\text { Klebsiella }(n=13)^{\# \#} \\
\text { Vitek }(n=13)\end{array}$} & \multicolumn{2}{|c|}{ Pseudomonas $(n=7)$} \\
\hline & Mini API $(n=12)$ & Vitek $(n=8)$ & & Mini API $(n=1)$ & Vitek $(n=6)$ \\
\hline Amoxicillin & 92 & 100 & 100 & 100 & 100 \\
\hline Ceftriaxone & 92 & 100 & 100 & 100 & 100 \\
\hline Ceftazidime & 92 & 87.50 & 92 & 0 & 67 \\
\hline Cefepime & 84 & 75 & 92 & 0 & 50 \\
\hline Amoxicillin + clavulanate & 84 & 100 & 100 & 100 & 100 \\
\hline Cefotaxime + clavulanate & 40 & 37.50 & 72 & 100 & 83 \\
\hline Ceftazidime + clavulanate & 40 & 37.50 & 54 & 100 & 67 \\
\hline Ticarcillin + clavulanate & 58 & NP & NP & 0 & NP \\
\hline Piperacillin + tazobactam & 50 & 50 & 77 & 0 & 33 \\
\hline Cefoperazone + sulbactam & NP & 12.50 & 62 & NP & 33 \\
\hline Cefepime + tazobactam & NP & 37.50 & 54 & $\mathrm{NP}$ & 33 \\
\hline Imipenem & 10 & 0 & 54 & 0 & 33 \\
\hline Meropenem & 10 & 12.50 & 70 & 0 & 33 \\
\hline Ertapenem & NP & NP & 62 & NP & NP \\
\hline Colistin & NP & 0 & 8 & 0 & 0 \\
\hline Tigecycline & NP & 0 & 54 & NP & 67 \\
\hline Gentamycin & 42 & 37.50 & 54 & 0 & 33 \\
\hline Amikacin & 30 & 12.50 & 46 & 0 & 33 \\
\hline Ciproflox & 92 & 87.50 & 77 & 0 & 33 \\
\hline Levoflox & 92 & 87.50 & 77 & 0 & 33 \\
\hline Nalidixic acid & 92 & 87.50 & 84 & NP & NP \\
\hline Nitrofurantoin & 58 & 70 & 84 & 100 & 83 \\
\hline Tetracycline & 75 & 75 & 84 & 100 & 83 \\
\hline Doxycycline & 75 & 75 & 84 & 100 & 83 \\
\hline Minocycline & 75 & 75 & 84 & 100 & 83 \\
\hline
\end{tabular}

Figures are in percentage; N.P: drug not in panel; ${ }^{\# \#}$ all the Klebsiella isolates were tested on Vitek 2. 
Staphylococcus was isolated in $38.6 \%(n=51)$ of cases and CONS in $4.5 \%$ of cases in the present paper. The isolation of Staphylococcus aureus is consistent with the study of Arora and Devi [17], Roy et al. [11], and Karlowsky et al. [13] where the reported isolation of the organism was $27.3 \%, 14 \%$, and $16.5 \%$, respectively. However, reported isolation of CONS was $20.16 \%, 16.5 \%$, and $42 \%$, respectively, in these studies which is quite higher than isolation of CONS seen in our study but in accordance with Anbumani et al. [12] where Staphylococcus aureus is reported as $36.4 \%$ and CONS as $1.12 \%$. Given that CONS isolated from blood are often skin contaminants which are clinically insignificant [1-5], we suspect that the observed low isolation of CONS in our paper could be due to or related to strict aseptic practices of collection method followed for blood sampling of blood culture. The burden of other Grampositive isolates was much lesser than Staphylococcus aureus which is in accordance with these studies.

Enterococcus was isolated in $3.8 \%(n=5)$ of cases. Out of these 4 were Enterococcus faecalis and 1 was Enterococcus gallinarum. Amongst the 4 Enterococcus faecalis 2 were vancomycin sensitive and 2 were vancomycin resistant, while Enterococcus gallinarum was moderately sensitive to vancomycin.

E. coli and Klebsiella (25\%) were the predominant Gramnegative isolates in our paper which is in accordance with other studies of Mehta et al. [7], Karlowsky et al. [13], Kamga et al. [9], and China and Gupta [8]. We also observed similar frequency of Pseudomonas and Acinetobacter as in these studies but we did not observe any isolate of Salmonella which is isolated in the frequency of 10 to $20 \%$ in these studies. Generally, Salmonella is community-acquired infection in general population which gains entry via feco-oral route. Since we cater to specific renal population, that might possibly be the causative factor for this observed difference of Salmonella.

We also observed that significant proportion of our patient pool is immunocompromised due to CKD status or postkidney transplantation status which led to bacteremia with various organisms like Burkholderia, Sphingomonas, Moraxella, Kocuria, and Micrococcus which commonly does not lead to bacteremia in healthy nonimmunocompromised individuals.

Staphylococcus aureus isolates in our study exhibited oxacillin resistance of $70.6 \%$ which is quite high from the studies of Kamga et al. [9], Kavitha et al. [10], China and Gupta, [8] and Karlowsky et al. [13] who reported a percentage of $18 \%, 40.8 \%, 49.5 \%$, and $29 \%$. This is in accordance with the studies of Garg et al. [6] who reported a percentage of $75.6 \%$. Vancomycin resistance in our Staphylococcus isolates was $21.6 \%$ which is in accordance with Kamga et al. [9] who recorded an isolation of $32 \%$. But it is in contrast to the studies of Karlowsky et al. [13], China and Gupta [8], Garg et al. [6], Kavitha et al. [10], and Roy et al. [11] who reported no resistance to vancomycin. The increasing glycopeptide resistance in our study could be due to widespread usage of the drug in the empirical treatment protocol of suspected CRBSI in dialysis population. However, the current resistance pattern emphasizes the importance of strict antibiotic policy to prevent emergence and spread of antibiotic resistance.
In view of significant oxacillin and vancomycin resistance of $70.6 \%$ and $21.6 \%$, respectively, in staphylococcal isolates, drugs like clindamycin, linezolid, daptomycin, and teicoplanin should be considered in the treatment of MRSA before vancomycin. Vancomycin resistant Enterococcus (VRE) in our study is $40 \%(2 / 5)$ which is in accordance with the studies like Garg et al. [6] and Karlowsky et al. [13] who reported $16.6 \%$ and $35.8 \%$, respectively.

Among the Gram-negative isolates, the Enterobacteriaceae isolates in our study showed very poor sensitivity to quinolones, penicillins, and cephalosporins. However combining BL+BLI did improve the sensitivity. Least resistance was observed with carbapenems, colistin, aminoglycosides, and tigecycline. We could not compare the sensitivity pattern of carbapenems, colistin, and tigecycline with other studies as these drugs were not tested in majority of the other studies.

ESBL producers detected in our study were 39.6\% which is in accordance with the study of Kavitha et al. [10] and Arora and Devi [17] who reported prevalence of ESBL producers as $32 \%$ and $34.4 \%$, respectively.

\section{Conclusion}

Staphylococcus aureus and organisms belonging to Enterobacteriaceae family are the leading causes of septicemia. The most sensitive drugs for Gram-positive isolates were tetracycline, teicoplanin, vancomycin, clindamycin, daptomycin, and linezolid and the most sensitive drugs for Gram-negative bacteria were carbapenems, colistin, aminoglycosides, and tigecycline. Clinicians should exercise caution in their use of vancomycin in order to preserve this useful antibiotic and prolong its therapeutic usefulness and replace its use by drugs like teicoplanin, daptomycin, and linezolid. Increasing incidence of drug resistant organisms like MRSA, VRE, and ESBL producers raises serious concerns about antibiotic resistance and mandates strict antibiotic policy on a large scale. As the practice of prescribing antibiotics is completely unregulated, cheap generics are available, usage of all kinds of antibiotics for even minor illnesses is widespread, and there are not many newer antimicrobials in research pipeline, it is foreseen that if the same kind of practice and scenario continues the antibiotic resistance is likely to go up and we will face serious crisis of antibiotics in near future.

\section{Conflict of Interests}

The authors declare that there is no conflict of interests regarding the publication of this paper.

\section{References}

[1] L. S. Young, "Sepsis syndrome," in Principle and Practice of Infectious Diseases, G. L. Mandell, J. E. Bennet, and R. Dolin, Eds., pp. 690-705, Churchill Livingstone, 1995.

[2] P. A. Fuselier, L. S. Garcia, G. W. Procop et al., "Blood stream infections," in Bailey and Scot's Diagnostic Microbiology, A. F. Betty, F. S. Daniel, and S. W. Alice, Eds., pp. 865-883, Mosby, 2002. 
[3] S. Trevini and C. R. Mahon, "Bacteraemia," in Textbook of Diagnostic Microbiology, R. M. Connie and G. Manusel, Eds., pp. 998-1008, WB Saunders, 2000.

[4] K. M. Elhag, A. K. Mustafa, and S. K. Sethi, "Septicaemia in a teaching hospital in Kuwait-I: incidence and aetiology," Journal of Infection, vol. 10, no. 1, pp. 17-24, 1985.

[5] M. Crowe, P. Ispahani, H. Humphreys, T. Kelley, and R. Winter, "Bacteraemia in the adult intensive care unit of a teaching hospital in Nottingham, UK, 1985-1996," European Journal of Clinical Microbiology and Infectious Diseases, vol. 17, no. 6, pp. 377-384, 1998.

[6] A. Garg, S. Anupurba, and J. Garg, "Bacteriological profile and antimicrobial resistance of blood culture isolates from a university hospital," Journal of Indian Academy of Clinical Medicine, vol. 8, no. 2, pp. 139-143, 2007.

[7] M. Mehta, P. Dutta, and V. Gupta, "Antimicrobial susceptibility pattern of blood isolates from a teaching hospital in North India," Japanese Journal of Infectious Diseases, vol. 58, no. 3, pp. 174-176, 2005.

[8] D. China and V. Gupta, "Bacteriological profile and antimicrobial susceptibility pattern of blood isolates from a tertiary care hospital in North India," International Journal of Pharmaceutical Research and Bioscience, vol. 2, no. 2, pp. 24-35, 2013.

[9] H. L. F. Kamga, A. L. Njunda, and P. F. Nde, "Prevalence of septicemia and antibiotic sensitivity pattern of bacterial isolates at the University Teaching Hospital, Yaoundae, Cameroon," African Journal of Clinical and Experimental Microbiology, vol. 12, no. 1, pp. 2-8, 2011.

[10] P. Kavitha, B. Sevitha, and R. Sunil, "Bacteriological profile and antibiogram of blood culture isolates in a pediatric care unit," Journal of Laboratory Physicians, vol. 2, pp. 85-88, 2010.

[11] I. Roy, A. Jain, M. Kumar, and S. K. Agarwal, "Bacteriology of neonatal septicemia in a tertiary care hospital of Northern India," Indian Journal of Medical Microbiology, vol. 20, pp. 156159, 2002.

[12] N. Anbumani, J. Kalyani, and M. Mallika, "Distribution and antimicrobial susceptibility of bacteria isolated from blood cultures of hospitalized patients in a tertiary care hospital," Indian Journal for the Practicing Doctor, vol. 5, no. 2, pp. 1-7, 2008.

[13] J. A. Karlowsky, M. E. Jones, D. C. Draghi, C. Thornsberry, D. F. Sahm, and G. A. Volturo, "Prevalence and antimicrobial susceptibilities of bacteria isolated from blood cultures of hospitalized patients in the United States in 2002," Annals of Clinical Microbiology and Antimicrobials, vol. 3, article 7, 2004.

[14] M. Mehdinejad, A. D. Khosravi, and A. Morvaridi, "Study of prevalence and antimicrobial susceptibility pattern of bacteria isolated from blood cultures," Journal of Biological Sciences, vol. 9, no. 3, pp. 249-253, 2009.

[15] M. Barati, M. T. Taher, R. Abasi, M. M. Zadeh, M. Barati, and A. R. Shamshiri, "Bacteriological profile and antimicrobial resistance of blood culture isolates," Iranian Journal of Clinical Infectious Diseases, vol. 4, no. 2, pp. 87-95, 2009.

[16] E. D. Ayobola, O. S. Egbule, and O. Omonigho, "Study of prevalence and antimicrobial susceptibility of blood culture bacterial isolates," Malaysian Journal of Microbiology, vol. 7, no. 2, pp. 78-82, 2011.

[17] U. Arora and P. Devi, "Bacterial profile of blood stream infections and antibiotic resistance pattern of isolates," JK Science, vol. 9, no. 4, pp. 186-190, 2007. 

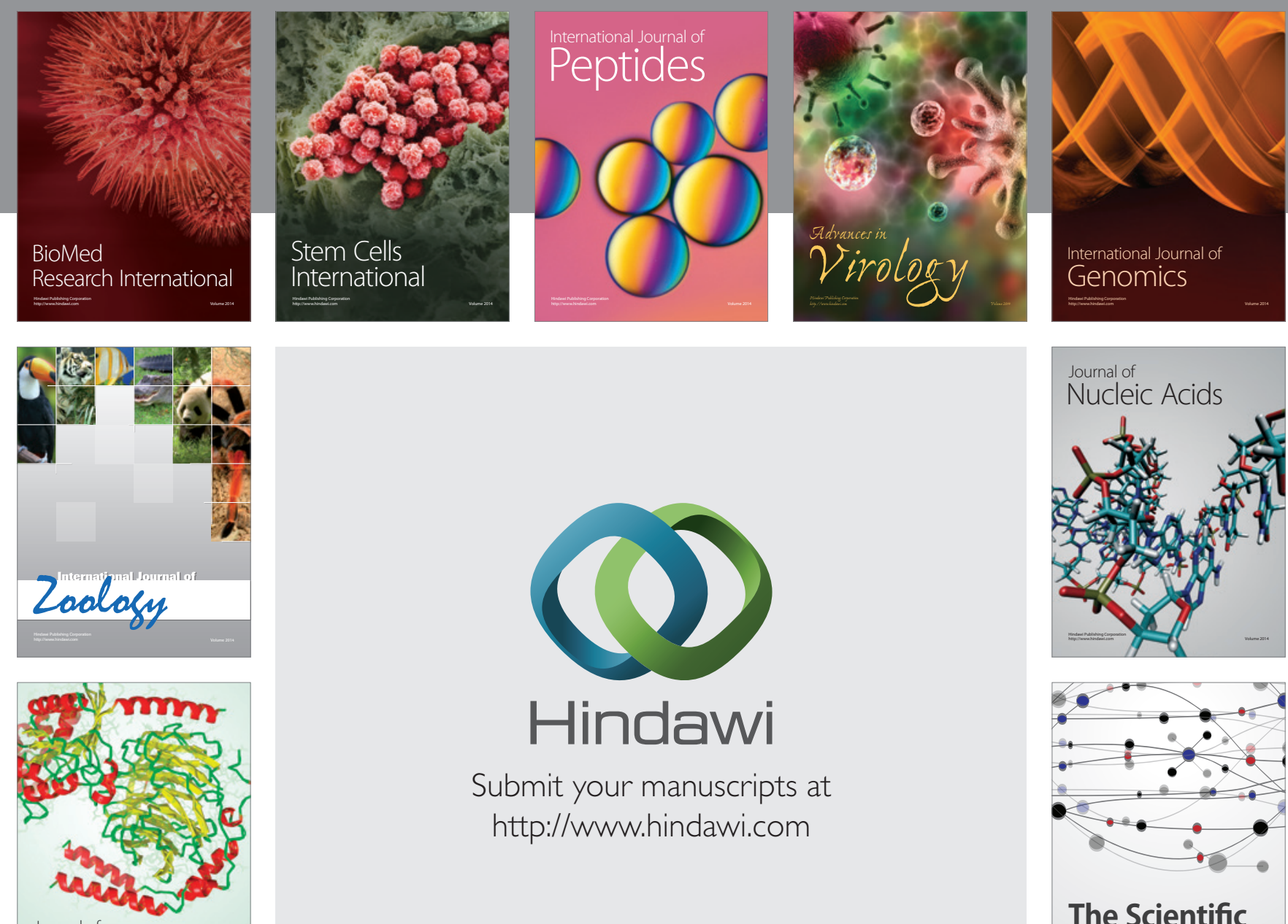

Submit your manuscripts at

http://www.hindawi.com

Journal of
Signal Transduction
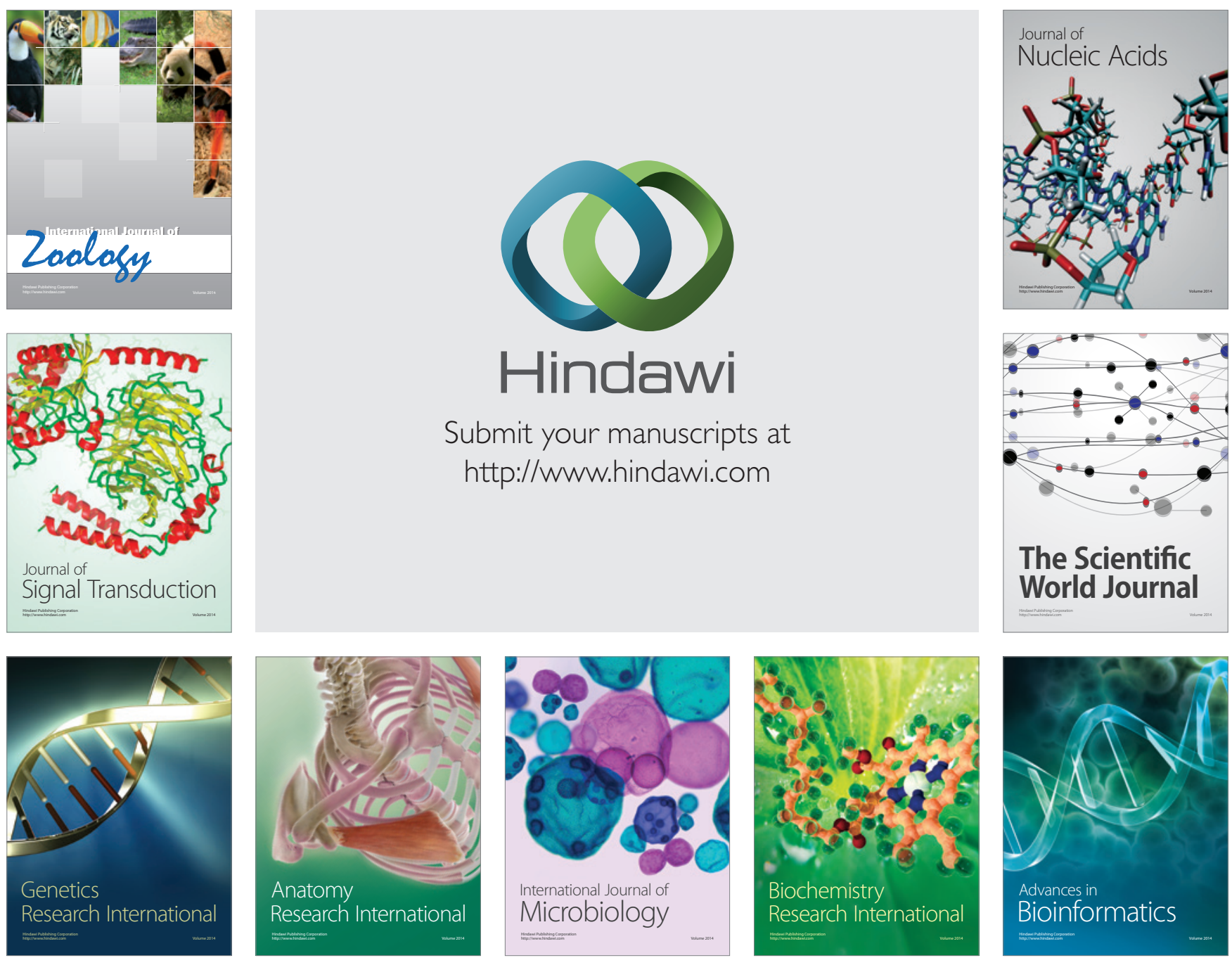

The Scientific World Journal
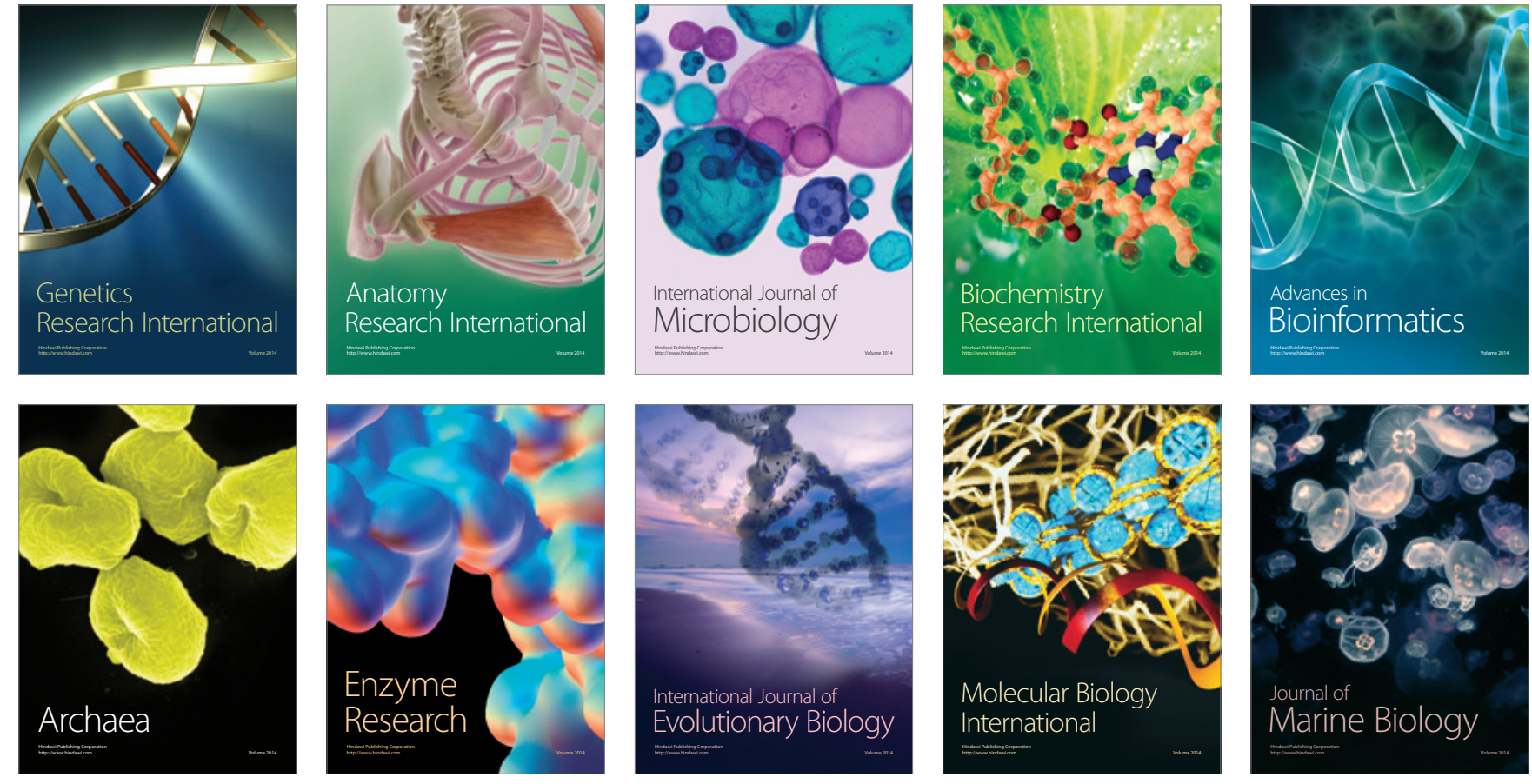